The numbers in parenthesis are powers of ten, e.g., $(-2) .33837=.33837 \times 10^{-2}$.

\begin{tabular}{rccc}
\hline$x$ & $F(x)$ & $\epsilon_{P}(x)$ & $\epsilon_{E}(x)$ \\
\hline 2 & $(-1) .57026$ & $(-3) .49$ & $(-2) .20$ \\
3 & $(-2) .33837$ & $(-3) .20$ & $(-5) .99$ \\
4 & $(-4) .79388$ & $(-4) .48$ & $(-4) .10$ \\
5 & $(-6) .71853$ & $(-5) .43$ & $(-5) .39$ \\
6 & $(-8) .24730$ & $(-5) .56$ & $(-5) .13$ \\
8 & $(-14) .15594$ & $(-5) .54$ & $(-7) .42$ \\
10 & $(-22) .19100$ & $(-5) .32$ & \\
\hline
\end{tabular}

For the range of values of $x$ given in the table the expression for $P$ is obviously more complicated than $E$ and, in fact, on a digital computer requires about thirty percent more computation time. Unfortunately, however, the worth of $E(x)$ is questionable for $x<2$, which is not the case for $P(x)$.

LTV Electrosystems, Inc.

Greenville, Texas

1. R. G. Hart, "A close approximation related to the error function," Math. Comp., v. 20, 1966, pp. 600-602. appear.)

2. H. L. GRAY, "A limiting case of the G-transformation," SIAM J. Numer. Anal. (To

3. H. L. Gray \& W. R. Schucany, "A new rational approximation to Mills' ratio," J. Amer. Statist. A ssoc. (To appear.)

4. C. Hastings, Approximations for Digital Computers, Princeton Univ. Press, Princeton, N. J., 1955, p. 167. MR 16, 963.

\title{
Improvement in Recurrence Techniques for the Computation of Bessel Functions of Integral Order
}

\author{
By Fr. Mechel
}

The Bessel functions satisfy recurrence relations which are very convenient for the generation of these functions, especially when a great number of functions with varying index is needed, [1], [2], [3].

To start with the spherical Bessel functions $j_{n}(x), y_{n}(x)$, the recurrence relation

$$
f_{n-1}(x)+f_{n+1}(x)=((2 n+1) / x) f_{n}(x)
$$

is valid, which can be used either in the upward direction of the index $n$ or in the downward direction. For the generation of the spherical Neumann functions $y_{n}(x)$ it must be used in the upward direction with the starting functions

$$
y_{0}(x)=-\cos x / x \text { and } y_{1}(x)=-\sin x / x-\cos x / x^{2} .
$$

The computation of the spherical Bessel function $j_{n}(x)$ with Eq. (1) is more difficult, since now the recurrence relation must be used in the downward direction.

Received February 17, 1967. 
According to the "state of the art" (see e.g. [3]) the computation starts with a "sufficiently high" index $N$ and enters with the trial functions

$$
\hat{j}_{N+1}=0, \quad \hat{j}_{N}=a,
$$

$a$ any small number, into the recurrence relation. By repeated use of Eq. (1) finally $\hat{j}_{0}$ is obtained. With the exact representation of $j_{0}(x)=\sin x / x$ a scale factor

$$
C=j_{0}(x) / \hat{j}_{0}(x)=\sin x / \hat{j}_{0} \cdot x
$$

is computed. The hopefully exact functions $j_{n}(x)$ follow from

$$
j_{n}(x)=C \cdot \hat{j}_{n}(x) \text {. }
$$

In a check run now the upper index $N$ is increased by a certain value and the computation is repeated. If $C$ is unaffected by the increase of $N$, the functions $j_{n}(x)$ are ready. Otherwise, the index $N$ must be further increased.

This method of the computation of $j_{n}(x)$ has several drawbacks:

(a) The determination of the upper index $N$ is awkward and time consuming.

(b) If $N$ was chosen too high and/or the initial value $a$ in Eq. (3) was too large, then number overflow of the computer will occur.

(c) By putting $\hat{j}_{N+1}=0$ in Eq. (3), an error in the "direction" of the recurrence relation is introduced besides the harmless scaling error. As a consequence of this starting error, a certain number of functions $j_{m}, j_{m+1}, \cdots, j_{N}$ at the upper index limit are useless because the error in these functions is too large. Even the number of these erroneous functions is unknown.

These difficulties are avoided by the following method.

The Neumann functions $y_{n}(x)$ are computed by ascending recurrence up to an index $N+1$ for which their order of magnitude surpasses a certain preassigned value $M$, that is $\left|y_{N+1}(x)\right| \geqq 10^{M},(M>4)$. The order of magnitude $M$ is a quantity which in most applications is imposed by the degree of numerical accuracy of the problem at hand. Then, with sufficient accuracy for these orders of magnitude, the relation

$$
j_{N}(x) \approx-1 /\left(x^{2} \cdot y_{N+1}(x)\right)
$$

follows from the cross product

$$
j_{n}(x) y_{n-1}(x)-j_{n-1}(x) y_{n}(x)=1 / x^{2} .
$$

We shall take the value of $j_{N}(x)$ according to Eq. (6) as the trial function $\hat{j}_{N}(x)$. For the evaluation of the trial function $\hat{j}_{N+1}$ we transform the recurrence relation (1) into

$$
g_{n}(x)=x \frac{j_{n-1}(x)}{j_{n}(x)}=(2 n+1)-\frac{x^{2}}{x j_{n}(x) / j_{n+1}(x)}
$$

that is,

$$
g_{n}(x)=(2 n+1)-x^{2} / g_{n+1}(x) .
$$

This leads to the continued fraction

$$
g_{n}(x)=(2 n+1)-\frac{x^{2}}{(2 n+3)-} \frac{x^{2}}{(2 n+5)-} \frac{x^{2}}{(2 n+7)-} \cdots
$$


It converges rather rapidly, provided $n>|x|$. A 15-decimal accuracy can be obtained by typically 10 fractions.

Computing $g_{N+1}(x)$ yields, together with $\hat{j}_{N}(x)$, the trial function $\hat{j}_{N+1}(x)$. Now the recurrence relation (1) is used in the downward direction, the scale factor is computed according to Eq. (4) and the functions $\hat{j}_{n}$ are corrected according to Eq. (5). By this method number overflows are avoided, the awkward guess of $N$ is replaced by a straightforward determination, the computation of the functions is performed up to a problem-oriented order of magnitude and the full accuracy is maintained up to the upper index limit $N$. The computation time for the calculation of the continued fraction $g_{N+1}(x)$ is more than compensated by the omission of the check of $N$ and by the fact that no surplus functions at the upper index limit have to be rejected for their lack of accuracy.

The generation of the functions $J_{n}(x)$ and $Y_{n}(x)$ is based upon the recurrence relation

$$
f_{n-1}(x)+f_{n+1}(x)=2 n f_{n}(x) / x .
$$

Again, it is used with increasing index $n$ for the computation of the Neumann functions $Y_{n}(x)$ and with decreasing $n$ for the computation of the Bessel functions $J_{n}(x)$. The starting functions $Y_{0}(x)$ and $Y_{1}(x)$ are obtained from their polynomial representation, [4] through [7]. The calculation of the functions $Y_{n}(x)$ with the help of Eq. (10) is continued up to $Y_{N+1}(x)$ with $\left|Y_{N+1}(x)\right| \geqq 10^{M}, M>4$. From the Wronskian

$$
J_{n+1}(x) Y_{n}(x)-J_{n}(x) Y_{n+1}(x)=2 / \pi x
$$

we take the approximation

$$
\hat{J}_{N}(x) \approx-(2 / \pi x)\left(1 / Y_{N+1}(x)\right)
$$

for one of the starting Bessel functions. With the function $G_{n}(x)$ defined by

$$
G_{n}(x)=x J_{n-1}(x) / J_{n}(x)
$$

the recurrence relation

$$
G_{n}(x)=2 n-x^{2} / G_{n+1}(x)
$$

is derived from Eq. (10). Again, $G_{N+1}(x)$ is computed by the continued fraction

$$
G_{n}(x)=2 n-\frac{x^{2}}{2 n+2-} \frac{x^{2}}{2 n+4-} \frac{x^{2}}{2 n+6-} \cdots
$$

which, together with $\hat{J}_{N}(x)$ from Eq. (12), yields the second starting function $\hat{J}_{N+1}(x)$ with which the recurrence relation (10) is entered in the downward direction. The scale factor $C$ is computed from

$$
C=\hat{J}_{0}(x)+2 \cdot \sum_{k=1}^{<N / 2>} \hat{J}_{2 k}(x)
$$

$\langle N / 2\rangle$ being the highest integer below or including $N / 2$. Eq. (16) is based upon the relation

$$
1=J_{0}(x)+2 \cdot \sum_{k=1}^{\infty} J_{2 k}(x)
$$


Very similar methods can be applied for the generation of the modified Bessel functions $I_{n}(x)$ and $K_{n}(x)$.

With the improvement just described the widely used recurrence techniques are very straightforward methods for the generation of the sets of Bessel functions with real argument $x$ and varying index $n$.

Institut für Angewandte Physik und Elektrotechnik

Universität des Saarlandes

Saarbrücken, Germany

1. I. A. Stegun \& M. Abramowitz, "Generation of Bessel functions on high speed computers," $M T A C$, v. 11,1957 , pp. 255-257. MR $20 \# 459$.

2. M. Goldstein \& R. M. Thaler, "Recurrence techniques for the calculation of Bessel functions," MTAC, v. 13, 1959, pp. 102-108. MR 21 \#4530.

3. M. Abramowitz \& I. A. STEgun, (EDITORS), Handbook of Mathematical Functions, with Formulas, Graphs, and Mathematical Tables, Dover, New York, 1966, pp. 386, 452.

4. C. W. Clenshaw, Chebyshev Series for Mathematical Functions, Nat. Phys. Lab. Math. Tables, Vol. 5, Dept. Scientific and Indust. Research, Her Majesty's Stationery Office, London, 1962. MR 26 \#362.

5. C. Hastings, Jr. \& J. P. Wong, Jr., "Analytical approximations," MT AC, v. 8, 1954, p. 46.

6. A. J. M. HITCHCOCK, "Polynomial approximations to Bessel functions of order zero and one and to related functions," $M T A C$, v. 11, 1957, pp. 186-188. MR 19, 64 .

7. J. WIMP, "Polynomial expansions of Bessel functions and some associated functions," Math. Comp., v. 16, 1962, pp. 446-458. MR $26 \# 6452$.

\section{The Zeros of $P_{\nu}^{1}(\cos \theta)$ and $\frac{\partial}{\partial \theta} P_{\mu}^{1}(\cos \theta)^{*}$}

\section{By Peter H. Wilcox}

Introduction. In the course of a recent study [1] of the scattering of an electromagnetic wave by a semi-infinite, perfectly conducting cone, it became necessary to compute numerically sets of positive zeros of certain associated Legendre functions treated as functions of their degree; that is, to find $\nu_{i}$ and $\mu_{i}, i=1,2,3, \cdots$, satisfying

$$
P_{\nu_{i}}^{1}(\cos \theta)=0
$$

and

$$
(\partial / \partial \theta) P_{\mu_{i}}^{1}(\cos \theta)=0,
$$

for a given $\theta$. The method presented here employs a trigonometric series expansion for the Legendre functions to obtain these zeros.

Formulas. An expression for the associated Legendre function valid for $0<\theta<$ $180^{\circ}$ is [2]

$$
\begin{aligned}
P_{\nu}^{\mu}(\cos \theta)= & \pi^{-1 / 2} 2^{\mu+1}(\sin \theta)^{\mu} \frac{\Gamma(\nu+\mu+1)}{\Gamma(\nu+3 / 2)} \\
& \cdot \sum_{k=0}^{\infty}\left\{\frac{(\mu+1 / 2)_{k}(\nu+\mu+1)_{k}}{k !(\nu+3 / 2)_{k}} \sin [(\nu+\mu+2 k+1) \theta]\right\} .
\end{aligned}
$$

Received January 11, 1967. Revised April 12, 1967.

* The research reported in this paper was sponsored by the Air Force Avionics Laboratory, Wright-Patterson Air Force Base, Ohio under Contract No. AF 33(615)-5170. 